\title{
Prevalence of hypertension in patients with trigeminal neuralgia
}

\author{
Antonia Teruel · Saravanan Ram • \\ Satish K. S. Kumar · Sepehr Hariri · \\ Glenn Thomas Clark
}

Received: 10 December 2008/ Accepted: 3 February 2009/Published online: 10 March 2009

(c) Springer-Verlag 2009

\begin{abstract}
It is unclear whether hypertension (HTN) is a predisposing factor for the development of trigeminal neuralgia (TN). The purpose of this study was to determine the prevalence of HTN in TN patients and controls at the USC Orofacial Pain and Oral Medicine Center. A retrospective chart review was conducted from a database of over 3,000 patient records from 2003 to 2007. We identified patients diagnosed with TN with or without HTN. A total of 84 patients ( 54 females; 30 males) between the ages of 33 and 93 years were diagnosed with TN; 37\% had TN with HTN and $32 \%$ of controls had HTN. The increased prevalence of HTN in the TN patients was not statistically significant $(P=0.50)$. Since, both TN and HTN are seen in the elderly, it is likely that HTN is simply a co-existing condition in patients with $\mathrm{TN}$ and not a risk factor for its development.
\end{abstract}

Keywords Hypertension - Idiopathic trigeminal neuralgia $\cdot$ Tic douloureux $\cdot$ Trigeminal neuralgia . Prevalence

\section{Introduction}

According to The International Headache Society (IHS), classical trigeminal neuralgia (TN) is defined as "a unilateral disorder characterized by brief electric shock-like

\footnotetext{
A. Teruel $(\bowtie) \cdot$ S. Ram · S. K. S. Kumar · G. T. Clark Orofacial Pain and Oral Medicine Center, USC School of Dentistry, 925 West 34th Street, Room 4333, Los Angeles, CA 90089-0641, USA

e-mail: teruel@usc.edu

S. Hariri

UCLA, Los Angeles, USA
}

pains, abrupt in onset and termination, limited to the distribution of one or more divisions of the trigeminal nerve". This is different from symptomatic TN, which is defined by IHS as "pain indistinguishable from classical $\mathrm{TN}$ but caused by a demonstrable structural lesion other than vascular compression" [1]. Classical $\mathrm{TN}$ is diagnosed based on history alone and clinical features of the pain. Patients often describe the pain as attacks or paroxysms, which may last for a few seconds to $2 \mathrm{~min}$. Interestingly between paroxysms there is a refractory period in which the pain cannot be triggered and the patient is asymptomatic. The intensity of the pain is severe and the quality is usually described as electric shock-like, sharp, stabbing, or shooting. The pain might be triggered spontaneously or by light touch in a specific area or simply by eating or talking. $\mathrm{TN}$ is considered to be a rare disease with an annual incidence of 5.9/100,000 women and 3.4/100,000 men in the USA. The incidence increases with age and tends to be higher in women at all ages with a male to female ratio of $2: 3$ [2].

The exact pathophysiology of TN is still unknown. In majority of TN cases the trigeminal nerve root entry zone has been found to be compressed by an aberrant loop of artery or vein, which ultimately leads to demyelination of the trigeminal nerve [3]. Furthermore, it has been demonstrated that $\mathrm{TN}$ is more common in patients with multiple sclerosis and an elevated relative risk has been associated with hypertension (HTN), particularly among women [2]. It has been suggested that patients with TN have arterial tortuosity, which may lead to increased arterial pulse pressure waveforms secondary to vascular stiffness [4]. However, very few studies have explored the relationship between HTN and TN [2, 4].

It is unclear if hypertension is a predisposing factor to the development of TN. Therefore, the objective of this 
study was to determine the prevalence of HTN in patients diagnosed with classical TN and describe the characteristics of classical TN including age, gender and race among the patient population seen at the Orofacial Pain and Oral Medicine Center at the USC School of Dentistry (USC OFP-OM Center) in Los Angeles, California, USA between June 2003 and August 2007.

\section{Materials and methods}

A retrospective chart review was conducted from the electronic medical record database (SOAPware, Fayetteville, AR) at USC OFP-OM Center of over 3,000 patient records from June 2003 to August 2007. The study was approved by the University of Southern California University Park Institutional Review Board and Ethics Committee (USC UPIRB \#UP-07-00416) and has therefore been performed in accordance with the ethical standards laid down in the 1964 Declaration of Helsinki. We identified all patients who were diagnosed with $\mathrm{TN}$ using the chart searcher function implemented in the SOAPware program with the appropriate search terminology. All patients were clinically diagnosed as having TN by the faculty, or residents under the supervision of faculty. A thorough history and head and neck exam was performed for every patient along with necessary radiographic investigations to rule out all potential dental and bony pathologies. Brain MRI with and without contrast was done for all patients prior to initiating treatment. Only patients with classic TN (idiopathic) without any obvious pathology such as multiple sclerosis, plaques, tumors, and abnormalities of the skull base were considered in our study. Inclusion criteria for TN included a history and clinical presentation that satisfied the International Headache Society criteria for classical TN [5].

Dental caries, periapical lesions, periodontal pockets with bone loss, cracked teeth, hyperocclusion, non-vital teeth and other bony pathologies were excluded with a thorough diagnostic workup. From this subset of TN patients, those currently taking anti-hypertensive medications for a minimum period of 6 months and with a diagnosis of HTN (established by the patient's physician) were considered as having both TN and HTN. For the control group we identified thrice the number of age- and gender-matched controls from the USC OFP-OM Center using the existing electronic medical record database (SOAPware ${ }^{\circledR}$ ). The controls were identified randomly by searching the database based on age and gender. Inclusion criteria were gender-matched and age-matched controls (matched within a 2-year range) who may or may not have a diagnosis of HTN. All control patients with HTN were diagnosed by their physician and were on anti-hypertensive medication.
Statistical analysis

All analyses were performed using Microsoft Excel $^{\circledR}$ spreadsheets and GraphPad InStat ${ }^{\circledR}$ software. Chi-square test with Yates correction and Fisher's exact test were performed to calculate the $P$ value.

\section{Results}

The study population comprised of a total of $84 \mathrm{TN}$ patients (54 female; 30 male) and age- and gender-matched controls ( $n=252 ; 162$ female; 90 male) between the ages of 33 and 93 years (mean 65.3 years). The racial characteristics of our patient population were as follows: Caucasian (102); Hispanic (117); Asian (33); Black (38); American Indian (3); Others (19) and Unknown (24).

Thirty-one patients with TN reported having HTN and were taking anti-hypertensive medication, out of which 13 of these patients were males and 18 were females. The prevalence of HTN in TN patients was 37\%. In the control population, 81 patients reported having HTN and were taking anti-hypertensive medication. Therefore, the prevalence of HTN in controls was $32 \%$. The percentage of cases and controls with HTN is shown in Fig. 1. The odds ratio of having $\mathrm{HTN}$ in $\mathrm{TN}$ is 1.24 (95\% confidence interval, 0.7-2). The difference in prevalence of HTN in TN cases versus controls was not found to be statistically significant using Chi-square test with Yates correction $(P=0.50)$ and Fisher's exact test $(P=0.42)$. Hispanics had the highest prevalence of HTN in both TN and controls followed by Caucasians.

\section{Discussion}

The risk factors that predispose an individual to develop TN include age and female gender. Although, arterial hypertension has been reported as a risk factor for developing TN based on the theory of increased arterial tortuosity and pulse pressure, little or no epidemiologic data exist to validate this concept. Arterial stiffness has been associated with the development of HTN [6] and its association with $\mathrm{TN}$ has been investigated, but this relationship has not been established since studies failed to demonstrate that patients with TN have an increase in arterial stiffness [4].

In a population-based study of TN patients conducted in Rochester, Minnesota, 25\% (19 out 75) of the patients with TN were found to have HTN with an odds ratio of 1.96 (95\% confidence interval, 1.2-3) [2]. In our study, we have found the prevalence of HTN in TN to be $37 \%$ compared to the $32 \%$ seen in the control population. The reported rates 


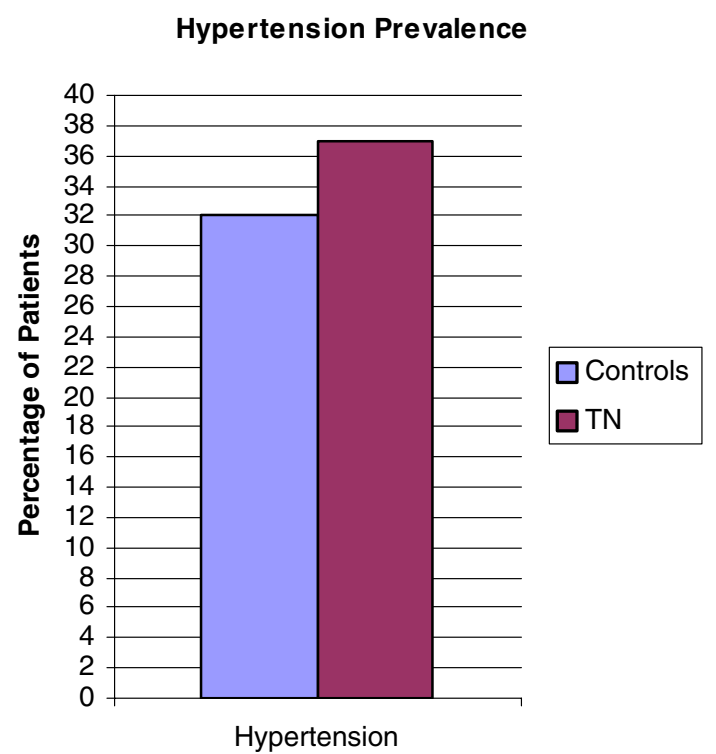

Fig. 1 Prevalence of HTN in cases versus controls; $X$ axis TN cases and controls; $Y$ axis Prevalence of HTN

of HTN in the normal population have ranged from 28.7 to $29.3 \%[7,8]$. The slight increase in the prevalence of HTN in the control group might be attributed to the fact that our sample is a convenience sample. The $5 \%$ difference in the prevalence of HTN between the two groups was not statistical significant. This might also be due to our sample size, which was too small to show a statistically significant difference. Also, $\mathrm{TN}$ is a rare disease, with an overall prevalence of $0.1-0.2$ per 1,000 and an incidence ranging from about 4-5/100,000/year [9], and therefore it is difficult to obtain a large TN patient sample. Larger populationbased studies might provide more evidence regarding the association between TN and HTN. In our study, HTN was not found to be a significant risk factor in patients with TN.

The limitations of our study include the following: (1) retrospective chart review, (2) convenience sample, which will restrict the extrapolation of prevalence data to general population, and (3) lack of data regarding the duration of HTN, which will be important as the vascular stiffness and nerve compression may worsen with prolonged hypertension. In general, retrospective studies are not the best way to study risk factors for diseases. Therefore, further prospective studies, with an accurate clinical assessment of HTN, are needed to conclusively clarify the possible relationship between HTN and TN.
To the best of our knowledge, there are no published studies on the racial differences in TN. Our data show that Hispanics had the highest prevalence of TN followed by Caucasians in comparison to other races. Since the Los Angeles area has a large population of Hispanics this could explain the higher prevalence of TN noted in Hispanics in this study. However, our study is comprised of a sample size with a very diverse racial composition, which prevents the racial differences from this study from being extrapolated to the general population of the United States.

In conclusion, our results suggest that there is no correlation between HTN and the development of TN. Since, both TN and HTN are seen in the elderly, it is possible that HTN is simply a co-existing condition in patients with TN. This conclusion should be taken with caution as this is a retrospective study and further prospective studies, with an accurate clinical assessment of hypertension, are needed to conclusively clarify the possible relationship between HTN and TN.

\section{Conflict of interest None.}

\section{References}

1. The International Classification of Headache Disorders. 2nd ed. (2004). Cephalalgia. 24 (Suppl 1): 1-160

2. Katusic S, Beard CM, Bergstralh E, Kurland LT (1990) Incidence and clinical features of trigeminal neuralgia, Rochester, Minnesota, 1945-1984. Ann Neurol 27:89-95

3. Devor M, Amir R, Rappaport ZH (2002) Pathophysiology of trigeminal neuralgia: the ignition hypothesis. Clin J Pain 18:4-13

4. Turner CL, Mendoza N, Illingworth RD, Kirkpatrick PJ (2003) Measurement of pulse pressure profiles in patients with trigeminal neuralgia. J Neurol Neurosurg Psychiatry 74:533-535

5. Oleson J (2004) The international classification of headache disorders. Cephalalgia 24:9-160

6. Arnett DK, Boland LL, Evans GW, Riley W, Barnes R, Tyroler HA, Heiss G (2000) Hypertension and arterial stiffness: the atherosclerosis risk in communities study. ARIC investigators. Am J Hypertens 13:317-323

7. Hajjar I, Kotchen TA (2003) Trends in prevalence, awareness, treatment, and control of hypertension in the United States, 19882000. JAMA 290:199-206

8. Ong KL, Cheung BM, Man YB, Lau CP, Lam KS (2007) Prevalence, awareness, treatment, and control of hypertension among United States adults 1999-2004. Hypertension 49:69-75

9. Manzoni GC, Torelli P (2005) Epidemiology of typical and atypical craniofacial neuralgias. Neurol Sci 26(Suppl 2):s65-s67 